\title{
An Interval Matrix Based Generalized Newton Method for Linear Complementarity Problems
}

\author{
Hai-Shan Han, Lan-Ying \\ College of Mathematics, Inner Mongolia University for the Nationalities, Tongliao, China \\ Email: haishanhan@sohu.com
}

Received 6 July 2015; accepted 9 August 2015; published 12 August 2015

Copyright (C) 2015 by authors and Scientific Research Publishing Inc.

This work is licensed under the Creative Commons Attribution International License (CC BY). http://creativecommons.org/licenses/by/4.0/

(c) (i) Open Access

\begin{abstract}
The penalty equation of LCP is transformed into the absolute value equation, and then the existence of solutions for the penalty equation is proved by the regularity of the interval matrix. We propose a generalized Newton method for solving the linear complementarity problem with the regular interval matrix based on the nonlinear penalized equation. Further, we prove that this method is convergent. Numerical experiments are presented to show that the generalized Newton method is effective.
\end{abstract}

\section{Keywords}

Linear Complementarity Problem, Nonlinear Penalized Equation, Interval Matrix, Generalized Newton Method

\section{Introduction}

The linear complementarity problem, denoted by $\operatorname{LCP}(M, q)$, is to find a vector $x \in R^{n}$ such that

$$
x \geq 0, \quad M x-q \geq 0, \quad x^{\mathrm{T}}(M x-q)=0
$$

where $M \in R^{n \times n}$ is a given matrix and $q \in R^{n}$ is a given vector. This problem serves as a unified formulation of linear and quadratic programming problems as well as of two-person (noncooperative) matrix-games, and has several important applications in economics and engineering sciences; see Cottle, Pang, and Stone [1] and its references. 
There exist several methods for solving $\operatorname{LCP}(M, q)$, such as projection method, multi splitting method, interior point method, and the nonsmooth Newton method, smoothing Newton method, homotopy method etc. See [1]-[6] and its references.

In [7], it given a nonlinear penalized Equation (1.2) corresponding to linear complementarity problem (1.1).

Find $x_{\lambda} \in R^{n}$ such that

$$
M x_{\lambda}+\lambda\left[x_{\lambda}\right]_{+}^{\frac{1}{k}}=q
$$

where $\lambda>1$ is the penalized parameter, $k>0,[u]_{+}=\max \{u, 0\}$ and $y^{\alpha}=\left(y_{1}^{\alpha}, y_{2}^{\alpha}, \cdots, y_{n}^{\alpha}\right)^{\mathrm{T}}$ for any $y=\left(y_{1}, y_{2}, \cdots, y_{n}\right)^{\mathrm{T}} \in R^{n}$.

The nonlinear penalized problems (1.2) corresponding to the linear complementarity problem (1.1), which its research has achieved good results. In 1984, Glowinski [1] studied nonlinear penalized Equation (1.2) in $R^{n}$, and proved the convergence of penalized equation that matrix A was symmetric positive definite. In 2006, Wang et al. [8] presented a power penalty function approach to the linear complementarity problem arising from pricing American options. It is shown that the solution to the penalized equation converges to that of the linear complementarity problem with matrix is positive definite. In 2008, Yang [7] proved that solution to this penalized Equations (1.2) converged to that of the LCP at an exponential rate for a positive definite matrix case where the diagonal entries were positive and off-diagonal entries were not greater than zero. The same year, Wang and Huang [9] presented a penalty method for solving a complementarity problem involving a secondorder nonlinear parabolic differential operator, and defined a nonlinear parabolic partial differential equation (PDE) approximating the variational inequality using a power penalty term with a penalty constant $\lambda>1$, a power parameter $k>0$ and a smoothing parameter $\varepsilon$. And prove that the solution to the penalized PDE converges to that of the variational inequality in an appropriate norm at an arbitrary exponential rate of the form $O\left(\left[\lambda^{-k}+\varepsilon\left(1+\lambda \varepsilon^{1 / k}\right)\right]^{1 / 2}\right)$. Under some assumptions, $\mathrm{Li}$ [10] [11] proved that the solution to this equation converges to that of the linear complementarity problem with $A$ is a strict row diagonally dominant upper triangular $P$-matrix when the penalty parameter approaches to infinity and the convergence rate was also exponential. It is worth mentioning that the penalty technique has been widely used solving nonlinear programming, but it seems that there is a limited study for the LCP.

Although the studies solving for the linear complementarity problem based on the nonlinear penalized equation have good results. But there is no method that is given for solving the nonlinear penalized equation. Throughout the paper, we propose a generalized Newton method for solving the nonlinear penalized equation with under the suppose $[M, M+\lambda I]$ is regular. So the method can better to solve linear complementarity problem. We will show that the proposed method is convergent. Numerical experiments are also given to show the effectiveness of the proposed method.

\section{Preliminaries}

Some words about our notation: $I$ refers to the identity matrix, and $\|\cdot\|$ represents the 2-norm. For $x \in R^{n}$, all vectors are column vectors, $x^{\mathrm{T}}$ refers to the transpose of the $x,[x]_{+}=\max \{x, 0\}$, that generalized Jacobian $\partial[x]_{+}=D(x)$, where $D(x)$ denotes diagonal matrix, On the diagonal elements with component 1.0 or $\sigma \in[0,1]$ corresponding to the component of $x$ which is positive, negative or zero, respectively.

The definition of interval matrix arises from the linear interval equations [12], given two matrices $\underline{A}=\left(\underline{A}_{i j}\right)$

and $\bar{A}=\left(\bar{A}_{i j}\right)$, an interval matrix $A^{I}=[\underline{A}, \bar{A}]=\{A: \underline{A} \leq A \leq \bar{A}\}$, where $\underline{A} \leq \bar{A}$ refers to $\underline{A}_{i j} \leq \bar{A}_{i j}$ for each $i, j . A^{I}$ is called regular if each $A \in A^{I}$ is nonsingular.

Lemma 1: Assume that interval matrix $[M, M+\lambda I]$ is regular. Then for diagonal matrix $D\left(x_{\lambda}\right)$ and real number $\lambda>1, M+\lambda D\left(x_{\lambda}\right)$ is nonsingular.

Proof: By definition of $D=\operatorname{diag}(x)$, for every x, we have $D \in[0, I]$ then 


$$
M+\lambda D \in[M, M+\lambda I]
$$

By the assumptions, we have $M+\lambda D\left(x_{\lambda}\right)$ is nonsingular.

Lemma 2: Let $x, y \in R^{n}$. Then

$$
\left\|[x]_{+}-[y]_{+}\right\| \leq\|x-y\|
$$

Definition 1 [1]: A matrix $M \in R^{n \times n}$ is said to be a P-matrix if all its principal minors are positive.

Lemma 3 [1]: A matrix $M \in R^{n \times n}$ is a P-matrix if and only if the $L C P(q, M)$ has a unique solution for all vectors $q \in R^{n}$.

\section{Generalized Newton Method}

In this section, we will propose that a new generalized Newton method based on the nonlinear penalized equation for solving the linear complementarity problem. Because when $k>1$, penalty term of the nonlinear penalized equation (1.2) is none Lipschitz continuously, hence we only discusses a case that $k=1$. So from nonlinear penalized equation (1.2), we have that

$$
M x_{\lambda}+\lambda\left[x_{\lambda}\right]_{+}=q
$$

These case penalty problems for the continuous Variational Inequality and the linear complementarity problems are discussed in [2] [13].

Let us note

$$
f\left(x_{\lambda}\right)=M x_{\lambda}+\lambda\left[x_{\lambda}\right]_{+}-q, \lambda>1
$$

Thus, nonlinear penalized equation (3.1) is equivalent to the equation $f\left(x_{\lambda}\right)=0$.

A generalized Jacobian $\partial f\left(x_{\lambda}\right)$ of $f\left(x_{\lambda}\right)$ is given by

$$
\partial f\left(x_{\lambda}\right)=M+\lambda D\left(x_{\lambda}\right)
$$

where $D\left(x_{\lambda}\right)=\partial\left[x_{\lambda}\right]_{+}$is a diagonal matrix whose diagonal entries are equal 1,0 or a real number $\sigma \in[0,1]$ depending on whether the corresponding component of $x_{\lambda}$ is positive, negative, or zero. The generalized Newton method for finding a solution of the equation $f\left(x_{\lambda}\right)=0$ consists of the following iteration:

$$
f\left(x_{\lambda}^{i}\right)+\partial f\left(x_{\lambda}^{i}\right)\left(x_{\lambda}^{i+1}-x_{\lambda}^{i}\right)=0
$$

Then

$$
\begin{gathered}
\partial f\left(x_{\lambda}^{i}\right) x_{\lambda}^{i+1}=\partial f\left(x_{\lambda}^{i}\right) x_{\lambda}^{i}-f\left(x_{\lambda}^{i}\right) \\
{\left[M+\lambda_{k} D\left(x_{\lambda}^{i}\right)\right] x_{\lambda}^{i+1}=\left[M+\lambda_{k} D\left(x_{\lambda}^{i}\right)\right] x_{\lambda}^{i}-\left[M x_{\lambda}^{i}+\lambda_{k}\left[x_{\lambda}^{i}\right]_{+}-q\right]} \\
=\lambda_{k} D\left(x_{\lambda}^{i}\right) x_{\lambda}^{i}-\lambda_{k}\left[x_{\lambda}^{i}\right]_{+}+q
\end{gathered}
$$

Since $D\left(x_{\lambda}^{i}\right) x_{\lambda}^{i}=\left[x_{\lambda}^{i}\right]_{+}$, consequently

$$
\left[M+\lambda_{k} D\left(x_{\lambda}^{i}\right)\right] x_{\lambda}^{i+1}=q
$$

Proposition $1 M x_{\lambda}+\lambda\left[x_{\lambda}\right]_{+}=q$ equivalent to $0 \leq z \perp A z+b \geq 0$, where

$$
z=M x_{\lambda}-q, \quad A=(M+\lambda I) M^{-1}, \quad b=\lambda M^{-1} q
$$

Proof: Since $\left|x_{\lambda}\right|+x_{\lambda}=\left\{\begin{array}{ll}2 x_{\lambda}, & x_{\lambda} \geq 0 \\ 0, & x_{\lambda}<0\end{array}\right.$, then $\left[x_{\lambda}\right]_{+}=\frac{1}{2}\left(\left|x_{\lambda}\right|+x_{\lambda}\right)$, we have 


$$
\begin{gathered}
M x_{\lambda}+\lambda\left[x_{\lambda}\right]_{+}-q=M x_{\lambda}+\lambda \frac{1}{2}\left(\left|x_{\lambda}\right|+x_{\lambda}\right)-q=\left(M+\lambda \frac{1}{2} I\right) x_{\lambda}+\lambda \frac{1}{2}\left|x_{\lambda}\right|-q=0 \\
(2 M+\lambda I) x_{\lambda}+\lambda\left|x_{\lambda}\right|-2 q=0
\end{gathered}
$$

By [14], its equivalent to

$$
\begin{gathered}
0 \leq(2 M+\lambda I) x_{\lambda}-\lambda x_{\lambda}-2 q \perp(2 M+\lambda I) x_{\lambda}+\lambda x_{\lambda}-2 q \geq 0 \\
0 \leq M x_{\lambda}-q \perp(M+\lambda I) x_{\lambda}-q \geq 0
\end{gathered}
$$

Let $z=M x_{\lambda}-q$ then $(M+\lambda I) x_{\lambda}-q=(M+\lambda I) M^{-1}(z+q)-q=(M+\lambda I) M^{-1} z+\lambda M^{-1} q$.

Proposition $2 M x_{\lambda}+\lambda\left[x_{\lambda}\right]_{+}=q$ has a unique solution if and only if the interval matrix $[M, M+\lambda I]$ is regular.

Proof: Since $M, M+\lambda I \in[M, M+\lambda I]$, by theorem 1.2 of [12], if $[M, M+\lambda I]$ is regular, then $(M+\lambda I) M^{-1}$ is P-matrix, which implies that the LCP has a unique solution for any $q \in R^{n}$ [1], from the relation between the (3.1) and the LCP (3.5), we can easily deduce that the (3.1) is uniquely solvable for any $q \in R^{n}$.

\section{Algorithm 3}

Step 1: Choose an arbitrary initial point $x_{\lambda_{0}}^{0} \in R^{n}, \varepsilon>0$ and given $\lambda_{0}>1, \mu>1, \sigma \in[0,1]$, let $k:=0$;

Step 2: for the $\lambda_{k}$, computer $x_{\lambda_{k}}^{i+1}$ by solving

$$
\left[M+\lambda_{k} D\left(x_{\lambda_{k}}^{i}\right)\right] x_{\lambda_{k}}^{i+1}=b
$$

Step 3: If $\left\|x_{\lambda_{k}}^{i+1}-x_{\lambda_{k}}^{i}\right\|<\varepsilon$, let $\bar{x}_{k}=x_{\lambda_{k}}^{i}$ go to step 4. Otherwise, $i=i+1$ go to step 2 .

Step4: If $\left\|\bar{x}_{k+1}-\bar{x}_{k}\right\|<\varepsilon$ and $\left\|M \bar{x}_{k}-q\right\|<\varepsilon$, terminate, $\bar{x}=\bar{x}_{k}$ is solution of LCP. Otherwise let $\lambda_{k+1}=\mu \lambda_{k}, \quad x_{\lambda_{k+1}}^{0}=\bar{x}_{k} \quad$ let $k:=k+1$, go to 2 .

\section{The Convergence of the Algorithm}

We will show that the sequence $\left\{x_{\lambda_{k}}^{i}\right\}_{i=1}^{\infty}$ generated by the generalized Newton iteration (3.6) converges to an accumulation point $\bar{x}_{k}$ associated with $\lambda_{k}$. First, we establish boundness of the sequence $\left\{x_{\lambda_{k}}^{i}\right\}$ for any $\lambda_{k}>0$ generated by the Newton iterates (3.6) and hence the existence of accumulation point at each generalized Newton iteration.

Theorem 3: Suppose that the interval matrix $\left[M, M+\lambda_{k} I\right]$ is regular. Then, the sequence $\left\{x_{\lambda_{k}}^{i}\right\}$ generated by Algorithm 3 is bounded. Consequently, there exits an accumulation points $\bar{x}_{k}$ such that $\left[M+\lambda_{k} D\left(\bar{x}_{k}\right)\right] \bar{x}_{k}=b$.

Proof: Suppose that sequence $\left\{x_{\lambda_{k}}^{i}\right\}$ is unbounded, Thus, there exists an infinite nonzero subsequence $\left\{x_{\lambda_{k}}^{i_{j}}\right\} \subset\left\{x_{\lambda_{k}}^{i}\right\}$ such that

$$
\left\{x_{\lambda_{k}}^{i_{j}}\right\} \rightarrow \infty, D\left(x_{\lambda_{k}}^{i_{j}}\right)=\tilde{D} \text { and } \tilde{D} \in[0, I]
$$

where $\tilde{D}$ is main diagonal element of diagonal matrix which is $1,0, \sigma \in[0,1]$. We know subsequence $\left\{\frac{x_{\lambda_{k}}^{i_{j}}}{\left\|x_{\lambda_{k}}^{i_{j}}\right\|}\right\}$ is bounded. Hence, exists convergence subsequence and assume that convergence point is $\tilde{x}$, and satisfy 


$$
\left(A+\lambda_{k} \tilde{D}\right) \frac{x_{\lambda_{k}}^{i_{j}}}{\left\|x_{\lambda_{i_{k}}}\right\|}=\frac{b}{\left\|x_{\lambda_{k}}^{i_{j}}\right\|}
$$

Letting $j \rightarrow \infty$ yields

$$
\left(M+\lambda_{k} \tilde{D}\right) \tilde{x}=0,\|\tilde{x}\|=1 .
$$

Since $M+\lambda_{k} \tilde{D} \in\left[M, M+\lambda_{k} I\right]$ and the interval matrix $\left[M, M+\lambda_{k} I\right]$ is regular, we know that $\left(M+\lambda_{k} \tilde{D}\right)^{-1}$ is exists and hence $\tilde{x}=0$, contradicting to the fact that $\|\tilde{x}\|=1$. Consequently, the sequence $\left\{x_{\lambda_{k}}^{i}\right\}$ is bounded and there exists an accumulation point $\bar{x}_{k}$ of $\left\{x_{\lambda_{k}}^{i}\right\}$ such that

$$
\bar{x}_{k}=\left(M+\lambda_{k} D\left(\bar{x}_{k}\right)\right)^{-1} b
$$

Under a somewhat restrictive assumption we can establish finite termination of the generalized Newton iteration at a penalized equation solution as follows.

Theorem 4: Suppose that the interval matrix $\left[M, M+\lambda_{k} I\right]$ is regular and $\left\|\left(M+\lambda_{k} D\left(x_{\lambda_{k}}^{i}\right)\right)^{-1}\right\|<\frac{1}{2 \lambda_{k}}$ holds for all sufficiently large $\lambda_{k}$, then the generalized Newton iteration (3.6) linearly converges from any starting point $x_{\lambda_{k}}^{0}$ to a solution $\bar{x}_{k}$ of the nonlinear penalized equation (3.1).

Proof: Suppose that $\bar{x}_{k}$ is a solution of nonlinear penalized equation. By the lemma $1, M+\lambda_{k} D\left(x_{\lambda_{k}}\right)$ is nonsingular. To simply notation, let $\bar{D}=D\left(\bar{x}_{k}\right), D^{i}=D\left(x_{\lambda_{k}}^{i}\right)$ and since $\left(M+\lambda_{k} D\left(\bar{x}_{k}\right)\right) \bar{x}_{k}=b$,

$$
\begin{aligned}
& {\left[M+\lambda_{k} D\left(x_{\lambda_{k}}^{i}\right)\right] x_{\lambda_{k}}^{i+1}=b \text { have } } \bar{D} \bar{x}_{k}=\left[\bar{x}_{k}\right]_{+}, \quad D^{i} x_{\lambda_{k}}^{i}=\left[x_{\lambda_{k}}^{i}\right]_{+} \text {, hence } \\
& M\left(x_{\lambda_{k}}^{i+1}-\bar{x}_{k}\right)=-\lambda_{k} D^{i} x_{\lambda_{k}}^{i+1}+b+\lambda_{k} \bar{D} \bar{x}_{k}-b=-\lambda_{k}\left(D^{i} x_{\lambda_{k}}^{i+1}-\left[\bar{x}_{k}\right]_{+}\right) \\
&=-\lambda_{k}\left(D^{i}\left(x_{\lambda_{k}}^{i+1}-x_{\lambda_{k}}^{i}+x_{\lambda_{k}}^{i}\right)-\left[\bar{x}_{k}\right]_{+}\right) \\
&=-\lambda_{k}\left(\left[x_{\lambda_{k}}^{i}\right]_{+}-\left[\bar{x}_{k}\right]_{+}\right)-\lambda_{k} D^{i}\left(x_{\lambda_{k}}^{i+1}-\bar{x}_{k}+\bar{x}_{k}-x_{\lambda_{k}}^{i}\right) \\
&=-\lambda_{k}\left(\left[x_{\lambda_{k}}^{i}\right]_{+}-\left[\bar{x}_{k}\right]_{+}\right)-\lambda_{k} D^{i}\left(\bar{x}_{k}-x_{\lambda_{k}}^{i}\right)-\lambda_{k} D^{i}\left(x_{\lambda_{k}}^{i+1}-\bar{x}_{k}\right) \\
&\left(M+\lambda_{k} D^{i}\right)\left(x_{\lambda_{k}}^{i+1}-\bar{x}_{k}\right)=-\lambda_{k}\left(\left[x_{\lambda_{k}}^{i}\right]_{+}-\left[\bar{x}_{k}\right]_{+}\right)-\lambda_{k} D^{i}\left(\bar{x}_{k}-x_{\lambda_{k}}^{i}\right) \\
&\left(x_{\lambda_{k}}^{i+1}-\bar{x}_{k}\right)=\left(M+\lambda_{k} D^{i}\right)^{-1}\left(-\lambda_{k}\left(\left[x_{\lambda_{k}}^{i}\right]_{+}-\left[\bar{x}_{k}\right]_{+}\right)+\lambda_{k} D^{i}\left(x_{\lambda_{k}}^{i}-\bar{x}_{k}\right)\right)
\end{aligned}
$$

since $\left\|\left(M+\lambda_{k} D\left(x_{\lambda_{k}}^{i}\right)\right)^{-1}\right\|<\frac{1}{2 \lambda_{k}}$ and by the lemma 2, we have

$$
\begin{aligned}
\left\|\left(x_{\lambda_{k}}^{i+1}-\bar{x}_{k}\right)\right\| & \leq\left\|\left(M+\lambda_{k} D^{i}\right)^{-1}\right\|\left(2 \lambda_{k}\left\|\left(x_{\lambda_{k}}^{i}-\bar{x}_{k}\right)\right\|\right) \\
& =2 \lambda_{k} \cdot\left\|\left(M+\lambda_{k} D^{i}\right)^{-1}\right\|\left\|\left(x_{\lambda_{k}}^{i}-\bar{x}_{k}\right)\right\|<\left\|\left(x_{\lambda_{k}}^{i}-\bar{x}_{k}\right)\right\|
\end{aligned}
$$

Letting $i \rightarrow \infty$ and taking limits in both sides of the last inequality above, we have

$$
\frac{\left\|\left(x_{\lambda_{k}}^{i+1}-\bar{x}_{k}\right)\right\|}{\left\|\left(x_{\lambda_{k}}^{i}-\bar{x}_{k}\right)\right\|}<1
$$

So the iteration (3.6) linearly converges to a solution $\bar{x}_{k}$ of nonlinear penalized equation (3.1). In here, we will focus on the convergence of Algorithm 3.

Theorem 5: Suppose M is P-Matrix and that the interval matrix $\left[M, M+\lambda_{k} I\right]$ is regular and 
$\left\|\left(M+\lambda_{k} D\left(x_{\lambda_{k}}^{i}\right)\right)^{-1}\right\|<\frac{1}{2 \lambda_{k}}$ holds, then Algorithm 3 linearly converges from any starting point $x_{\lambda_{0}}^{0}$ to a solution $x^{*}$ of the $\operatorname{LCP}(M, q)(1.1)$.

Proof: Since M is P-Matrix, then the $\operatorname{LCP}(M, q)$ has a unique solution, let the solution denote $x^{*}$, by the assumptions of the theorem, the generalized Newton iteration (3.6) linearly converges to a solution $\bar{x}_{k}$ of the nonlinear penalized equation (3.1).

$$
\left\|\bar{X}_{\lambda_{k+1}}-x^{*}\right\|=\left\|\bar{X}_{\lambda_{k+1}}-x_{\lambda_{k+1}}^{i}+x_{\lambda_{k+1}}^{i}-x_{\lambda_{k}}^{i}+x_{\lambda_{k}}^{i}-x^{*}\right\| \leq\left\|\bar{X}_{\lambda_{k+1}}-x_{\lambda_{k+1}}^{i}\right\|+\left\|x_{\lambda_{k+1}}^{i}-x_{\lambda_{k}}^{i}\right\|+\left\|x_{\lambda_{k}}^{i}-x^{*}\right\|
$$

let $i \rightarrow \infty$, then

$$
\left\|\bar{X}_{\lambda_{k+1}}-x^{*}\right\| \leq\left\|\bar{X}_{\lambda_{k+1}}-\bar{x}_{\lambda_{k}}\right\|+\left\|\bar{X}_{\lambda_{k}}-x^{*}\right\|
$$

we have $\lim _{k \rightarrow \infty} \frac{\left\|\bar{x}_{\lambda_{k+1}}-x^{*}\right\|}{\left\|\bar{x}_{\lambda_{k}}-x^{*}\right\|} \leq 1$

\section{Numerical Experiments}

In this section, we give some numerical results in order to show the practical performance of Algorithm 2.1. Numerical results were obtained by using Matlab R2007 (b) on a 1G RAM, 1.86 Ghz Intel Core 2 processor. Throughout the computational experiments, the parameters were set as $\varepsilon=1.0 e-8, \lambda_{0}=10, \mu=2$.

Example 1: The matrix $A$ of linear complementarity problem $\operatorname{LCP}(A, b)$ of as follows (This example appear in the Geiger and Kanzow [15], Jiang and Qi [16], YONG Long-quan, DENG Fang-an, CHEN Tao [17]):

$$
A=\left(\begin{array}{cccccc}
4 & -1 & 0 & \cdots & 0 & 0 \\
-1 & 4 & -1 & \cdots & 0 & 0 \\
0 & -1 & 4 & \cdots & 0 & 0 \\
\vdots & \vdots & \vdots & \ddots & \vdots & \vdots \\
0 & 0 & 0 & \cdots & 4 & -1 \\
0 & 0 & 0 & \cdots & -1 & 4
\end{array}\right), b=(-1,-1, \cdots,-1,-1)^{\mathrm{T}}
$$

The computational results are shown in Table 1 . This $x^{0}$ is initial point, $k$ is number of inner iterations, the outer iteration number is $m, x^{*}$ is iteration results.

Example 2: The matrix $A$ of linear complementarity problem $\operatorname{LCP}(A, b)$ of as follows (This example appear in the Geiger and Kanzow [15], Jiang and Qi [16], YONG Long-quan, DENG Fang-an, CHEN Tao [17]):

$$
A=\operatorname{diag}\left(\frac{1}{n}, \frac{2}{n} \cdots, 1\right), b=(-1,-1,-1, \cdots, 1,-1)^{\mathrm{T}}
$$

The computational results are shown in Table 2. This $x^{0}$ is initial point, $k$ is number of inner iterations, the outer iteration number is $m, x^{*}$ is iteration results.

Table 1. Result from example 1.

\begin{tabular}{ccccc}
\hline$n$ & $x^{0}$ & $k$ & $m$ & $x^{*}=\left(\bar{x}_{1}, \bar{x}_{2}, \cdots, \bar{x}_{n}\right)^{\mathrm{T}}$ \\
\hline $\mathbf{6}$ & $(0,0,0,0,0,0)^{\mathrm{T}}$ & $\mathbf{3}$ & $\mathbf{2}$ & $(-0.3659,-0.4634,-0.4878,-0.4878,-0.4634,-0.3659)^{\mathrm{T}}$ \\
$\mathbf{6}$ & $(-2,0,-2,-2,0,-2)^{\mathrm{T}}$ & $\mathbf{3}$ & $\mathbf{2}$ & $(-0.3659,-0.4634,-0.4878,-0.4878,-0.4634,-0.3659)^{\mathrm{T}}$ \\
$\mathbf{7}$ & $(0,0,0,0,0,0,0)^{\mathrm{T}}$ & $\mathbf{3}$ & $\mathbf{2}$ & $(-0.3659,-0.4639,-0.4896,-0.4948,-0.4896,-0.4639,-0.3660)^{\mathrm{T}}$ \\
$\mathbf{7}$ & $(1,1,1,1,1,1,1)^{\mathrm{T}}$ & $\mathbf{3}$ & $\mathbf{2}$ & $(-0.3659,-0.4639,-0.4896,-0.4948,-0.4896,-0.4639,-0.3660)^{\mathrm{T}}$ \\
\hline
\end{tabular}


Table 2. Result from example 2.

\begin{tabular}{cccccc}
\hline$n$ & $x^{0}$ & $k$ & $m$ & $x^{*}=\left(\bar{x}_{1}, \bar{x}_{2}, \cdots, \bar{x}_{n}\right)^{\mathrm{T}}$ \\
\hline $\mathbf{6}$ & $(1,-1,1,-1,1,-1)^{\mathrm{T}}$ & $\mathbf{3}$ & $\mathbf{2}$ & $(-6,-3,-2,-1.5,-1.2,-1)^{\mathrm{T}}$ \\
$\mathbf{6}$ & $(0,0,0,0,0,0)^{\mathrm{T}}$ & $\mathbf{3}$ & $\mathbf{2}$ & $(-6,-3,-2,-1.5,-1.2,-1)^{\mathrm{T}}$ \\
$\mathbf{8}$ & $(0,0,0,0,0,0,0,0)^{\mathrm{T}}$ & $\mathbf{3}$ & $\mathbf{2}$ & $(-8,-4,-2.67,-2,-1.6,-1.34,-1.14,-1)^{\mathrm{T}}$ \\
$\mathbf{8}$ & $(1,-1,1,-1,1,-1,1,-1)^{\mathrm{T}}$ & $\mathbf{3}$ & $\mathbf{2}$ & $(-8,-4,-2.67,-2,-1.6,-1.34,-1.14,-1)^{\mathrm{T}}$ \\
$\mathbf{1 6}$ & $(1,-1,1,-1, \cdots, 1,-1)^{\mathrm{T}}$ & $\mathbf{3}$ & $\mathbf{2}$ & $(-16,-8,-5.3,-4,-3.2,-2.67,-2.28,-2,-1.78,-1.6,-1.45,-1.34,-1.23,-1.14,-1.06,-1)^{\mathrm{T}}$ \\
$\mathbf{1 6}$ & $(0,0,0,0, \cdots, 0,0)^{\mathrm{T}}$ & $\mathbf{3}$ & $\mathbf{2}$ & $(-16,-8,-5.3,-4,-3.2,-2.67,-2.28,-2,-1.78,-1.6,-1.45,-1.34,-1.23,-1.14,-1.06,-1)^{\mathrm{T}}$ \\
\hline
\end{tabular}

\section{Supported}

This work supported by the Science Foundation of Inner Mongolia in China (2011MS0114)

\section{References}

[1] Cottle, R.W., Pang, J.-S. and Stone, R.E. (1992) The Linear Complementarity Problem. Academic Press, San Diego

[2] Glowinski, R. (1984) Numerical Methods for Nonlinear Variational Problems. Springer-Verlag, New York. http://dx.doi.org/10.1007/978-3-662-12613-4

[3] Kanzow, C. (1996) Some Non-Interior Continuation Methods for Linear Complementarity Problem. SIAM Journal on Matrix Analysis and Applications, 17, 851-868. http://dx.doi.org/10.1137/S0895479894273134

[4] Facchinei, F. and Pang, J.S. (2003) Finite-Dimensional Variational Inequalities and Complementarity Problems. Springer, New York.

[5] Han, J.Y., Xiu, N.H. and Qi, H.D. (2006) Nonlinear Complementarity Theory and Algorithms. Shanghai Science and Technology Publishing House, Shanghai. (In Chinese)

[6] Murty, K.G. (1988) Linear Complementarity, Linear and Nonlinear Programming. Heldermann, Berlin.

[7] Wang, S. and Yang, X.Q. (2008) Power Penalty Method for Linear Complementarity Problems. Operations Research Letters, 36, 211-214. http://dx.doi.org/10.1016/j.orl.2007.06.006

[8] Wang, S., Yang, X.Q. and Teo, K.L. (2006) Power Penalty Method for a Linear Complementarity Problems Arising from American Option Valuation. Journal of Optimization Theory and Applications, 129, 227-254. http://dx.doi.org/10.1007/s10957-006-9062-3

[9] Wang, S. and Huang, C.S. (2008) A Power Penalty Method for Solving a Nonlinear Parabolic Complementarity Problem. Nonlinear Analysis, 69, 1125-1137. http://dx.doi.org/10.1016/j.na.2007.06.014

[10] Li, Y., Han, H.S., Li, Y.M. and Wu, M.H. (2009) Convergence Analysis of Power Penalty Method for Three Dimensional Linear Complementarity Problem. Intelligent Information Management Systems and Technologies, 5, 191-198.

[11] Li, Y., Yang, D.D. and Han, H.S. (2012) Analysis to the Convergence of the Penalty Method for Linear Complementarity Problems. Operations Research and Management Science, 21, 129-134. (In Chinese)

[12] Rohn, J. (1989) Systems of Linear Interval Equation. Linear Algebra and its Application, 126, 39-78.

[13] Bensoussan, A. and Lions, J.L. (1978) Applications of Variational Inequalities in Stochastic Control. North-Holland, Amsterdam, New York, Oxford.

[14] Mangasarian, O.L. and Meyer, R.R. (2006) Absolute Value Equations. Linear Algebra and Its Applications, 419, 359-367. http://dx.doi.org/10.1016/j.laa.2006.05.004

[15] Geiger, C. and Kanzow, C. (1996) On the Resolution of Monotone Complementarity Problems. Computational Optimization and Applications, 5, 155-173. http://dx.doi.org/10.1007/BF00249054

[16] Jiang, H.Y. and Qi, L.Q. (1997) A New Nonsmooth Equations Approach to Nonlinear Complementarity Problems. SIAM Journal on Control and Optimization, 45, 178-193. http://dx.doi.org/10.1137/S0363012994276494

[17] Yong, L.Q., Deng, F.A. and Chen, T. (2009) An Interior Point Method for Solving Monotone Linear Complementarity Problem. Journal of Mathematics, 29, 681-686. (In Chinese) 